Canadian Journal of Regional Science

Revue canadienne des sciences régionales

\title{
Analyse des mobilisations autour des sentiers de Balagne (Corse), l'innovation territoriale par les pratiques de loisirs de nature en question
}

\section{Hélène Melin}

Volume 44, numéro 2, 2021

Innovations et territoires face aux inégalités

Soumis : 11 mars 2020

Accepté : 3 mai 2021

URI : https://id.erudit.org/iderudit/1083326ar

DOI : https://doi.org/10.7202/1083326ar

Aller au sommaire du numéro

Éditeur(s)

Canadian Regional Science Association / Association canadienne des sciences régionales

\section{ISSN}

0705-4580 (imprimé)

1925-2218 (numérique)

Découvrir la revue

\section{Citer cet article}

Melin, H. (2021). Analyse des mobilisations autour des sentiers de Balagne (Corse), l'innovation territoriale par les pratiques de loisirs de nature en question. Canadian Journal of Regional Science / Revue canadienne des sciences régionales, 44(2), 12-22. https://doi.org/10.7202/1083326ar
Résumé de l'article

Cet article se base sur l'analyse d'une recherche ethnologique menée entre 2014 et 2018 en Balagne, sur le littoral nord-ouest de la Corse. Après avoir analysé les mobilisations habitantes et les politiques publiques locales portant sur les sentiers de proximité, nous nous demanderons si les chemins ruraux peuvent constituer un " bien commun " mobilisateur pour enclencher une dynamique territoriale vertueuse, à la fois respectueuse de l'environnement et des habitants. La dynamique collective observée peut-elle être analysée comme un processus d'innovation territoriale visant un développement « juste " et intégré d'un espace écologiquement fragile et soumis à une pression anthropique forte? 


\title{
CANADIAN JOURNAL OF REGIONAL SCIENCE \\ REVUE CANADIENNE DES SCIENCES REGIONALES
}

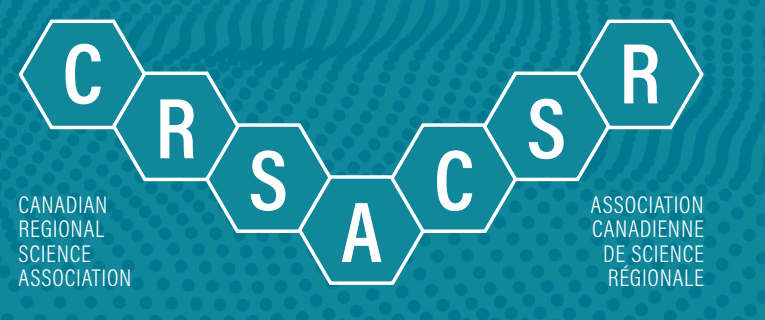

\section{ANALYSE DES MOBILISATIONS AUTOUR DES SENTIERS DE BALAGNE (CORSE), L'INNOVATION TERRITORIALE PAR LES PRATIQUES DE LOISIRS DE NATURE EN QUESTION}

\section{Hélène Melin}

\author{
Hélène Melin \\ Maître de conférences en sociologie et anthropologie \\ Laboratoire CLERSE-CNRS UMR 8019 \\ Faculté des sciences économiques, sociales et des territoires \\ Université de Lille \\ Campus Cité Scientifique \\ Avenue Paul Langevin \\ 59655 Villeneuve d'Asq cedex \\ France \\ Helene.melin@univ-lille.fr
}

Soumis: 11 mars 2020

Accepté : 3 mai 2021

Résumé: Cet article se base sur l'analyse d'une recherche ethnologique menée entre 2014 et 2018 en Balagne, sur le littoral nordouest de la Corse. Après avoir analysé les mobilisations habitantes et les politiques publiques locales portant sur les sentiers de proximité, nous nous demanderons si les chemins ruraux peuvent constituer un «bien commun » mobilisateur pour enclencher une dynamique territoriale vertueuse, à la fois respectueuse de l'environnement et des habitants. La dynamique collective observée peut-elle être analysée comme un processus d'innovation territoriale visant un développement «juste » et intégré d'un espace écologiquement fragile et soumis à une pression anthropique forte?

Mots Clés: attachement, communs, écologie, identité, politiques publiques, sentiers 
répondre à cela dans un contexte de compétitivité accrue entre microrégions? La dynamique constatée autour des sentiers pédestres témoigne-t-elle d'un renouvellement de la gouvernance locale, permettant d'inclure les habitants et d'adapter les politiques locales aux spécificités culturelles et naturelles des lieux de vie?

adossées, apparaissent aujourd'hui sur différents territoires comme des outils «en vogue » pour favoriser ou impulser un développement territorial, dans un contexte plutôt rural. Ces parcours sont régulièrement présentés comme des supports de loisirs de nature «respectueux», car le plus souvent pédestres, basés sur la découverte du patrimoine naturel et culturel et permettant une redynamisation des petits commerces et artisanats locaux (Blasquiet-Revol, 2017). Plusieurs études ont montré l'impact positif des loisirs de nature sur l'évolution des politiques d'aménagement, et les sentiers comme des instruments privilégiés des politiques publiques locales (HascharNoé, 2009). Cependant, les recherches restent marginales et éclatées entre différents champs disciplinaires (Boutelet, 1983; Etcheverria, 1997; Haschar-Noé, 2009; Sahuc, 2010). Les impacts de cette forme de dynamisation territoriale semblent également sous-étudiés. Les risques sont présents et méritent d'être questionnés: accentuation des pressions sur le milieu naturel, déchets, piétinements, conflits d'usages (Torre et al., 2006), questionnement sur la qualité de vie (Maupertuis et al., 2016), etc. Notre cas d'étude ${ }^{1}$ se situant en milieu insulaire, ces problématiques y sont d'autant plus aigües (Logossah \& Maupertuis, 2007).

Theys a défini en 2007 les inégalités écologiques comme une «forme spécifique d'inégalité sociale qui concerne soit l'exposition aux pollutions ou aux risques, soit l'accès à la nature ou aux aménités urbaines ou rurales» (Theys, 2007: 25). Dans les années suivantes s'est engagé un débat nourri concernant la dénomination à adopter, entre «inégalités écologiques» et «inégalités environnementales». La définition de Theys a été reprise pour qualifier les inégalités environnementales et c'est au final cette dernière terminologie qui s'est imposée, comme le montre Deldrève (2020), dans la lignée des travaux de Pye et al. (2008). Les inégalités environnementales comprennent à la fois des dimensions objectives et des aspects « ressentis ». C'est en ce sens que Deldrève reprend la définition de Charles: «ce qui peut se mesurer et s'objectiver» et «l'appréhension subjective des dynamiques de l'action » (Deldrève, 2015: 15). Dans la suite de l'article nous retiendrons donc cette expression, d'autant qu'elle marque la dimension locale, contextuelle et directement vécue au quotidien des inégalités et que nos propos sont appliqués à un cas d'étude particulier. Nous retiendrons aussi que ces inégalités témoignent, par rapport aux populations concernées, de leur «capacité d'agir pour leur environnement et de bénéficier des politiques qui leur sont dédiées » (Claeys et al., 2016: 73). Cependant, nous adhérons également à la notion complémentaire d'inégalités écologiques formulée par Bourg, inégalités envisagées comme globales, caractérisant notamment «le basculement des écosystèmes vers un état inconnu et hostile» (Bourg, 2020: 26). Cette approche permet en effet d'élargir la «communauté de destin » face aux problèmes environnementaux et donc de sortir d'une approche trop anthropocentrée, pour privilégier une approche écocentrée qui tienne compte de l'ensemble des composantes des écosystèmes.

Dans cette réflexion sur les inégalités environnementales et écologiques, les sentiers peuvent être envisagés comme des points d'appui, non seulement en termes d'aménagements, mais plus fondamentalement de relation au territoire, de construction de l'appartenance aux lieux et aux cultures qui s'y adossent. II s'agit de les considérer comme des indices qualitatifs d'une identification territoriale productrice de sens et d'engagement environnemental. Nous tenterons de comprendre quels rôles jouent les sentiers de proximité dans un processus d'innovation territoriale visant un développement «juste» et intégré d'un espace écologiquement fragile et soumis à une pression anthropique forte. Pour les gestionnaires, comment

L'étude du cas des mobilisations sociales et politiques engagées en Balagne doit permettre d'analyser ce que signifient les sentiers et ce qu'ils permettent de révéler sur le territoire (I). Nous tenterons d'identifier les valeurs sous-jacentes à la pratique des sentiers situés aux abords des villages et des hameaux, tant pour des habitants que de la part des acteurs institutionnels et des élus locaux. Les valeurs mobilisées par les différents intervenants semblent relever à la fois d'un registre de l'attachement - culturel, social et symbolique - à ses composantes écosystémiques et d'un registre du "réalisme», relevant du pragmatisme économique. II nous faut comprendre dans quelle mesure ces approches peuvent se compléter ou si elles s'opposent et si ces interactions permettent une construction territoriale innovante. II apparaît qu'à travers la diversité des approches, c'est avant tout le dialogue - faisant consensus ou dissensus - et le partage des usages comme du projet politique, qui peuvent amener une gouvernance écologique, durable et renouvelée (II).

\section{UNE DYNAMIQUE SOCIALE ET POLITIQUE COMMUNE EN BALAGNE: LA RÉOUVERTURE DES SENTIERS RURAUX COMME MARQUEURS DE L'IDENTITÉ DU TERRITOIRE}

Traiter, à travers l'étude des sentiers, les rôles possibles des traces laissées par les humains au sein de leur territoire de vie dans une dynamique écologique et sociale couplée, suppose de s'intéresser aux liens noués entre une communauté de vivants et son biotope (Londei, 2020). Cela présuppose qu'il existe des interrelations fortes, durables, de l'ordre des échanges matériels et des échanges idéels, entre les espèces et les composantes du milieu. Suivant en cela les propos de Descola (2011) et de Kohn (2017), l'approche adoptée souhaite élargir « la communauté du vivant » à l'ensemble des éléments signifiants qui expliquent les modes de vie et l'organisation du territoire. Autrement dit, les sentiers sont étudiés comme des composantes à part entière de la communauté, pas simplement des outils, mais des traces matérielles et sociales, qui explicitent les relations aux lieux. II s'agit de les envisager comme des «biens communs », au sens donné par Descola qui évoque le sens premier des communs: «non pas tant d'une ressource dont l'exploitation serait ouverte à tous, que d'un milieu partagé dont chacun est comptable» (Descola, 2015: 19). C'est également souligner que les chemins dont il est question ici sont à considérer dans leur matérialité mais également dans ce qu'ils renvoient comme mode de relations au milieu. Dans ce cadre, les cheminements sont à envisager comme des supports de l'action collective, des lieux producteurs de sens et des manifestations d'une identité locale partagée, à la fois naturelle et culturelle.

L'approche ethnologique semble particulièrement adaptée à la compréhension des processus à l'œuvre, relevant à la fois d'usages particuliers d'une nature familière et de reconstructions politiques et sociales basées sur l'histoire du territoire. Les sentiers sont en effet saisis dans une relation affective aux lieux, une dimension sensible (Sansot, 2000), et dans un rapport plus pragmatique d'aménagement durable du territoire. La méthode d'investigation qui est la nôtre est donc inductive. Nous avons couplé « une sociologie du regard et du cheminement » (Viard, 2012: 20) et une ethnologie de l'expérience et du vécu, telle que prônée par Moscovici (2002). L'analyse présentée ici repose sur une enquête qualitative menée durant quatre ans. Elle

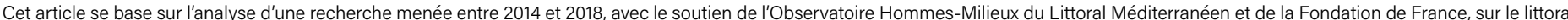
nord-ouest de la Corse. 
s'est traduite par la mobilisation de l'observation participante et non participante, via l'arpentage quotidien des sentiers en mode dynamique et en mode statique (en cumulé, environ 120 jours d'observations). L'enquête s'est aussi faite à travers des entretiens non directifs et semi-directifs auprès d'usagers des sentiers et des acteurs institutionnels (100 entretiens ont été réalisés). La démarche a été complétée par la réalisation d'un atelier d'échange collectif entre habitants et gestionnaires et la construction d'un court questionnaire à visée exploratoire ${ }^{2}$. Comme l'a souligné Mathieu (2016), enquêter sur les liens à la «nature» - ici via les sentiers - demande du temps et de la connivence avec les enquêtés. L'enquête par immersion était indispensable pour découvrir les usages effectifs des sentiers au-delà des discours souvent spontanés sur le rejet de pratiques associées aux touristes.

\section{Le contexte corse}

La Corse est une île méditerranéenne septentrionale de $8680 \mathrm{~km}^{2}$ comptant un peu plus de 340000 habitants $^{3}$. Collectivité territoriale unique française depuis 2018, ce statut lui confère une assemblée délibérante et un champ d'action large pour la gestion de son territoire. Elle est dirigée depuis 2015 par une coalition nationaliste ${ }^{4}$ qui a mis dans ses priorités les questions environnementales et de développement durable.
Les questions du développement et de l'écologie sont apparues très tôt sur le territoire. Les élus corses s'en sont saisi dans les années 1960-70, à travers des mesures pour protéger le patrimoine naturel (Fabiani, 2018). C'est ainsi qu'a été créé un Parc naturel régional en 1972 et un Conseil des rivages en 1975 (Martinetti, 2007). Très rapidement, les écologistes sont alliés aux nationalistes. Les années 2000 marquent l'«ambition d'un développement local reposant sur l'environnement et l'identité » (Lefevre, 2001: 48). Pour autant, il ne semble pas y avoir d'unanimité autour de la politique de préservation de l'île pendant de nombreuses années, avec des élus qui rejettent une gestion et des directives par l'État français, pour faire valoir la spécificité corse et son besoin de développement. Martinetti souligne la complexité de la situation corse, entre une « identité culturelle indéniable » (Martinetti, 2007: 29), un «capital naturel» à préserver et un débat contradictoire sur l'adaptation du tourisme à l'insularité. II y a aussi des atermoiements dans la mise en place d'une politique d'aménagement spécifique, cristallisés par la difficulté à faire émerger un Plan d'aménagement et de développement durable corse (PADDUC) (Tafani, 2010).

La Balagne (figure 1), notre terrain d'étude, est une microrégion rurale, structurée en Pays depuis $2003^{5}$, d'une superficie de $900 \mathrm{~km}^{2}$, située au nord-ouest de la Corse. Le territoire a connu une mutation d'usages et d'occupation importante après la Seconde Guerre

Figure 1. La Balagne, une microrégion rurale avec une façade littorale très touristique

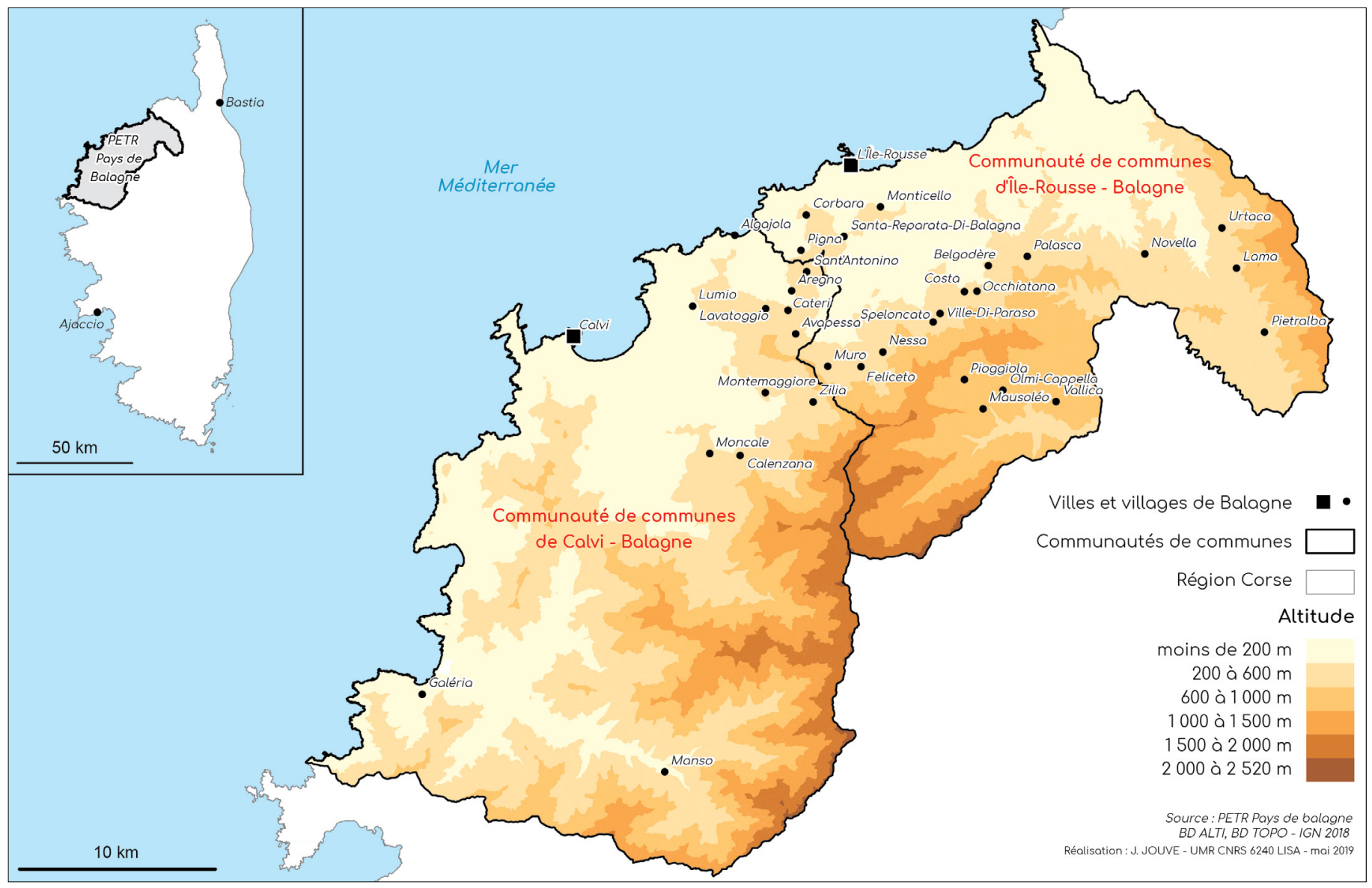

2 Voir supra et note de bas de page numéro 6

3 Données INSEE, 2017.

4 Le président du conseil exécutif est Gilles Siméoni, parti Femu a Corsica et le président de l'assemblée est Jean-Guy Talamoni du parti Corsica Libera.

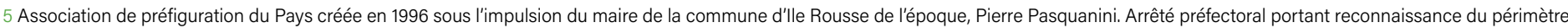
définitif du Pays de Balagne en novembre 2003. 
mondiale (Tafani, 2010). Les années 1950-60 voient une remise en question forte des valeurs portées par la société corse. L'économie jusqu'alors villageoise, centrée sur l'autonomie, l'autosuffisance productive et alimentaire, ainsi qu'un modèle d'existence porté par une organisation familiale et une hiérarchie politique locale via des «chefs de clans» (Lenclud, 2012) sont bouleversés par l'arrivée de populations nouvelles (Fabiani, 2018) et de nouvelles orientations économiques et de développement territorial portées par l'État français. Terre agricole au fort potentiel touristique, la Balagne est devenue aujourd'hui la première destination touristique de Corse (Tafani, 2010). Si les paysages montrent encore actuellement de façon prégnante la vocation horto-pastorale du territoire, force est de constater que la dynamique économique et foncière est portée par un tourisme balnéaire estival (Vuillamier, 2019). Ce «retournement» du territoire, comme cela est souvent évoqué, n'est pas sans poser de questions quant à la durabilité des processus engagés, l'évolution des modes de vie et le bien-être habitant, ainsi que l'équilibre entre empreinte et emprise humaines et bon état écologique des milieux naturels (Melin, 2015).

\section{Les sentiers de Balagne, une histoire culturelle et naturelle ancienne}

La Balagne, de par ses caractéristiques naturelles, culturelles et socioéconomiques, apparait comme un territoire d'étude pertinent pour analyser les possibilités d'innovation territoriale, tant en termes de gouvernance et de coopération entre acteurs de la société civile, que pour questionner l'évolution des liens entre espèces et milieux dans une optique d'écologisation des sociétés (Kalaora, 2010).

II s'agit d'un Pays à l'identité marquée, qui présente une histoire longue des chemins, renforcée par le caractère insulaire corse ainsi que son histoire politique.

Le caractère insulaire du territoire a en effet des répercussions sur l'environnement naturel et, par rebond, sur les sentiers. Les pressions exercées sur les milieux, du fait en particulier de la très forte fréquentation touristique, sont d'autant plus prégnantes que l'espace est limité et isolé. Pression écologique et foncière, dépendance économique au secteur des services autour du tourisme, autant de faits qui questionnent la dynamique collective constatée autour des sentiers. Dans ce cadre, traiter de l'innovation autour d'un processus d'aménagement des chemins est nécessairement complexe et mobilise plusieurs cadres théoriques. Inventer, à travers les chemins, de nouvelles manières de parcourir et d'habiter les lieux apparait ici comme un motif d'engagement local (Dechézelles \& Olive, 2016), car au-delà d'une pratique de loisirs, il s'agit de repenser l'agencement du territoire, son mode d'occupation et sa vocation, tant sociale que politique et économique.

Les sentiers en Balagne sont très anciens, liés à la vocation agricole du territoire (Lenclud, 2012) et ont été utilisés de façon quotidienne jusqu'aux années 1960. La disparition progressive de l'activité agricole et des modes de vie traditionnels, suite à «une évolution conjoncturelle due à l'ouverture des marchés économiques, des vagues d'incendie qui voient disparaître les oliveraies et châtaigneraies, et un exode rural» (Melin, 2015: 5), entraine l'abandon de la plupart des sentiers. La dimension anthropologique du chemin comme «composante de la matrice culturelle » (Etcheverria 1997: 11) semble alors disparaître de façon inexorable.

À partir des années 1990 cependant, les sentiers font l'objet d'un regain d'intérêt, comme cela a pu être le cas sur différents territoires ruraux en quête d'attractivité nouvelle (Perrin-Malterre, 2014). L'avènement des activités de pleine nature (Ginelli, 2017) couplé à un dé- sir de «reconnexion » au vivant participe également à un retour des sentiers, tant de la part d'associations excursionnistes que de gestionnaires d'espaces naturels (Mignotte, 2002). En Balagne littorale est également posée la question du rééquilibrage de la fréquentation touristique entre bords de mer et arrière-pays. S'il s'agit là d'un point clé des politiques publiques locales, cette diversification des usages apparaît aussi chez les visiteurs occasionnels. "Au désir du spectacle de la mer s'ajoute celui de la rencontre avec le pays», note Kalaora en 2010 (2010: 63). Même si en Corse, on continue «à venir massivement pour la mer» (Fabiani, 2018: 97), l'enquête a montré une demande des touristes pour des circuits patrimoniaux ${ }^{6}$.

\section{Le réinvestissement des sentiers balanins: des dynamiques sociales et politiques parallèles}

Autrefois fréquentés pour des usages majoritairement liés aux pratiques professionnelles et à des sociabilités de proximité, les sentiers sont aujourd'hui avant tout un objet de plaisir, tant pour les habitants que pour les visiteurs. Leur redécouverte s'est en effet opérée autour de la promotion de la randonnée. Pratique excursionniste exogène, la marche sportive sur les sentiers corses a d'abord été associée au tourisme, à l'étranger venu en quête d'exploit sportif. Fortement critiqué et pourtant perçu comme inéluctable, le développement touristique est envisagé, selon Martinetti, comme «une aliénation » (2007: 36) par une majorité de la population résidente.

Les critiques liées à la présence d'excursionnistes extérieurs au territoire montrent un décalage entre les pratiques habitantes et les usages importés. Depuis le début des années 2000 cependant, des pratiques autochtones se développent. Grandes randonnées et trails sont en vogue localement. L'analyse que font Lenclud (2012) et Fabiani (2018) des modes de sociabilité corses ainsi que des attachements au territoire appuie la thèse d'un écart entre les usages récréatifs exogènes et les pratiques endogènes. Cela ne veut pour autant pas dire que les Corses ignorent la beauté de leur pays ou qu'ils n'arpentent pas les lieux pour le plaisir. Comme des études sur les milieux de montagne l'ont montré, les habitants ont de tout temps eu une perception plurielle de leurs lieux de vie, à la fois matérielle, esthétique, concrète et symbolique. Chamboredon (1985) a bien montré les usages différents, voire opposés, de la nature rurale. Comme les habitants du grand narbonnais rencontrés par Claeys-Mekdade et Jacqué, les Corses apprécient la nature, en particulier de proximité: «Elle renvoie en effet à des sensations, des états ressentis comme le bien-être, le calme, la tranquillité. (...) Elle fait partie de l'ordre du familier» (Claeys-Mekdade \& Jacqué, 2000: 21). Pourtant, la Corse en général et la Balagne en particulier se distinguent d'autres territoires touristiques sur la question des usages et de leur légitimité. Outre les anciennes rivalités fortes entre communautés villageoises de montagne et communautés littorales (Lenclud, 2012) qui peuvent en partie expliquer l'attrait limité de nombreux Corses pour les loisirs de bord de mer, il semble avoir existé de tout temps en Corse un rejet des normes imposées, en particulier via les institutions administratives et les découpages territoriaux. Lenclud a constaté un rejet des façons de faire des administrateurs venus du continent. Cela se reporte sur les pratiques de loisirs, popularisées par des urbains (Ginelli, 2017) et amenées en Corse via le tourisme et les Corses exilés, qui imposent d'autres normes que celles qui ont cours, par « infiltration interne» (Lenclud, 2012). Fabiani a par ailleurs analysé que la relation historique au pouvoir français a conduit les Corses à intérioriser une distance vis-à-vis de la culture française et en parallèle à «forcer les traits de leur insularité » (Fabiani, 2018: 5), ce qui peut se traduire par un rejet à priori des pratiques récréatives de l'Hexagone.

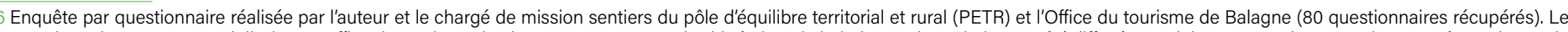

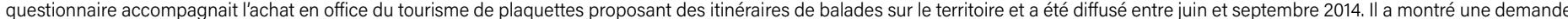

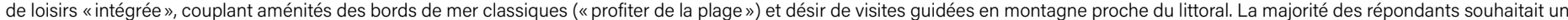
binôme mer-montagne et nature-découverte du patrimoine culturel durant leur séjour. 
En dehors d'une fréquentation touristique, des pratiques locales prenant appui sur les sentiers de proximité, situés en bordure littorale et autour des communes, ont existé par le passé, elles se renouvellent et se diversifient aujourd'hui. Les sentiers concernés relèvent de la voirie des collectivités territoriales ${ }^{7}$. II s'agit des chemins ruraux appartenant au domaine privé des communes, des chemins vicinaux et de certains chemins d'exploitation agricole, ou encore de chemins du domaine privé de l'État, tels les chemins forestiers gérés par l'Office national des forêts (ONF). En Balagne, tous les sentiers valorisés par les acteurs institutionnels et les habitants relèvent de ces statuts. II s'agit de chemins préexistant à leur mise en tourisme et appartenant à l'histoire des lieux, contrairement à certains réseaux de sentiers créés en Corse du Sud ex-nihilo pour promouvoir un tourisme vert et servir d'appui à l'éducation à l'environnement ${ }^{8}$.

Une politique territoriale volontariste et des intervenants multiples Le Pays de Balagne, pôle d'équilibre territorial et rural (PETR), regroupe l'ensemble des communes de la microrégion, réparties sur

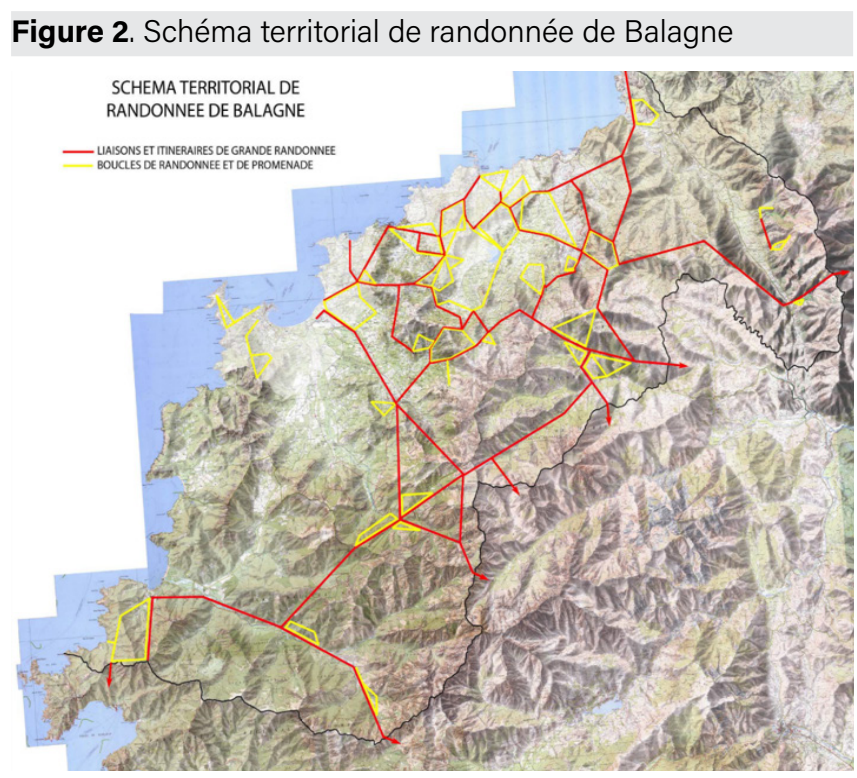

Source: Pays de Balagne

Figure 3. Réseau de boucles de randonnées de la Communauté de communes Lisula Balagna

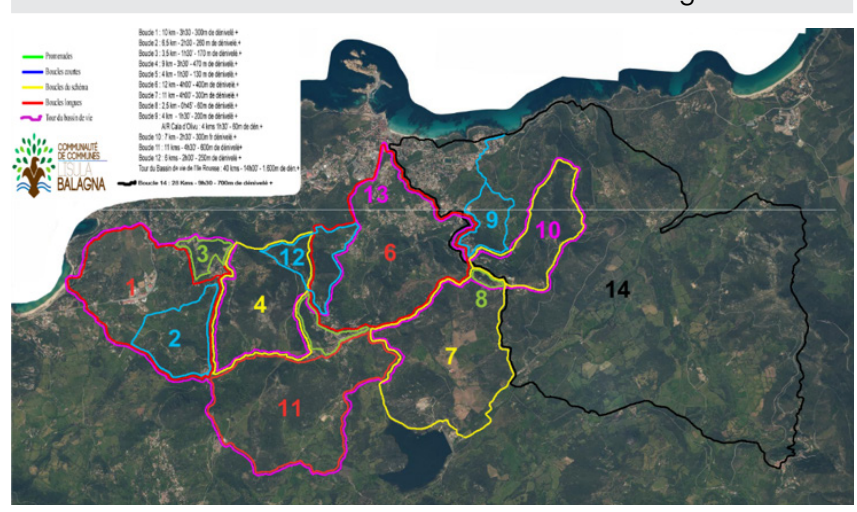

Source: Communauté de communes Lisula Balagna deux intercommunalités: la Communauté de communes (CC) de l'île-Rousse - Balagne (Lisula Balagna) et la Communauté de communes de Calvi-Balagne. Entre 2003 et 2012, le syndicat mixte, devenu PETR, engage une politique d'identification des anciens sentiers pour la mise en place d'un réseau d'itinéraires et de boucles de promenades accessibles au plus grand nombre (figure 2). La CC Lisula Balagna double ce premier réseau en instaurant ses propres circuits (figure 3).

Parallèlement, plusieurs communes créent leur propre réseau de chemins. D'autres acteurs institutionnels interviennent également sur la même période. Le Conservatoire du littoral balise et aménage des portions du sentier littoral, avec le conseil départemental qui a délégation d'entretien. La Direction départementale des territoires et de la mer (DDTM) de Haute-Corse entreprend de fixer les limites du domaine public maritime (DPM) le long du littoral, pour assurer la servitude de passage piéton le long du rivage. Au niveau régional, l'Office de l'environnement de la Corse (OEC) crée pour sa part un label «Sentiers du patrimoine» et identifie deux boucles de promenades en Balagne et une en limite de territoire nord. En limite sud du Pays, l'ONF gère les sentiers de la réserve naturelle du Fango.

Les objectifs affichés de cette politique menée à plusieurs niveaux sont doubles. II s'agit d'enclencher un processus de développement durable en encourageant un tourisme responsable, dirigé vers la découverte patrimoniale, l'arrière-pays littoral et déployé sur l'année plutôt qu'en période estivale. II s'agit également d'encourager les habitants à réinvestir leurs lieux de vie par des pratiques de mobilité douce.

Des habitants impliqués, qui créent leurs propres parcours Alors que les Balanins semblaient avoir déserté les chemins de façon assez brutale dans les années 1970, des pratiques redémarrent et se réinventent depuis une vingtaine d'années (Melin \& Poli, 2021). II s'agit d'une part de pratiques sportives exogènes réappropriées par les résidents permanents. D'autre part, des pratiques autochtones sont ravivées à travers des usages de proximité relevant de la «déambulation» (Le Breton, 2000). Des associations sportives et culturelles se créent pour organiser des sorties sur des sentiers qu'elles balisent et entretiennent pour l'occasion. Une association environnementale, I Sbuleca Mare, prend appui sur les sentiers littoraux pour faire de la sensibilisation écologique et organise plusieurs fois par an, avec des habitants volontaires, des campagnes d'arrachage de "griffes de sorcière», une plante invasive en bord de mer, qui se développe notamment de part et d'autre des chemins et vient concurrencer la végétation locale.

À côté de ces usages organisés, des habitants de plus en plus nombreux se promènent, seuls, en famille ou en groupes restreints, sur les chemins qui se situent autour de leurs lieux de vie, au besoin en réouvrant eux-mêmes des tracés disparus sous le maquis. Si l'enquête menée n'a pas cherché à établir un profil sociodémographique strict des usagers, ni à établir un échantillon représentatif, privilégiant plutôt une démarche «flottante», il est cependant possible de caractériser les enquêtés rencontrés. Corses d'origine en majorité, continentaux installés de façon permanente ${ }^{9}$ ensuite, il s'agit de retraités pour une moitié d'entre eux. Les autres se distribuent entre 25 et 65 ans, hommes et femmes à équivalence, leurs professions sont diverses, on sort ici clairement de ce qui serait une fréquentation de classe (Kalaora, 1993). Les facteurs liés à la pratique des sentiers apparaissent plus «qualitatifs », liés aux histoires familiales, aux lieux, à l'attachement aux composantes du territoire et à la volonté

Ordonnance $n^{\circ}$ 59-115 du 7 janvier 1959 relative à la voirie des collectivités locales. Version consolidée au 23 juin 1989.

8 DOCOB Natura 2000, 2017, site de Campumoru Senetosa.

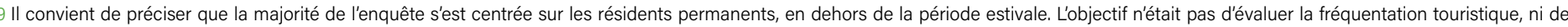

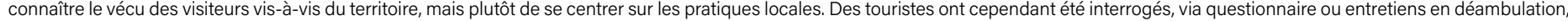
pour compléter la connaissance des usages des sentiers. 
de les préserver. Comme l'ont montré Deldrève et al, pour différentes luttes, en Balagne «la variable socio-économique apparait dès lors, presque secondaire (...) et articulée à d'autres catégories telles que l'autochtonie, l'ethnie, la « race »... » (Deldrève et al., 2019: 11). Effectivement, la notion d'autochtonie, même si elle n'apparait pas explicitement dans les discours, semble une variable de poids dans la définition et la défense des usages de la nature. Elle est alors envisagée, à la suite de Papinot, «avant tout comme un rapport social où le fait d'être reconnu comme étant 'vraiment d'ici' compose une ressource mobilisable» (cité par Ginelli, 2017: 36).

Les sentiers pratiqués par ces autochtones ne sont alors pas balisés ni forcément connus au-delà d'un cercle restreint d'habitués et témoignent d'un processus de réappropriation qui relève de l'attachement et du registre d'une relation sensible et affective aux lieux. Ces promenades réactivent dans le même temps des usages traditionnels, invisibilisés par une vision des chemins comme aménités environnementales réservées aux loisirs. Un savoir naturaliste local ancien se réinvente. II s'agit ici d'usages plus «fondamentaux» que le simple arpentage, montrant un lien de type totémiste (Descola, 2011) au milieu. Ces usages s'ancrent dans des pratiques de cueillette de plantes aromatiques et médicinales, des relevés naturalistes et d'un intérêt porté aux composantes de l'écosystème. Les sentiers sont alors le support d'une identification collective culturelle au territoire, qui illustre un mode de vie proche de la nature et des connaissances en matière de biodiversité pour protéger les milieux.

\section{UNE CONSTRUCTION TERRITORIALE CHEMIN FAISANT... INNOVER ET AGIR POUR LA JUSTICE ENVIRONNEMENTALE PAR LES PARADOXES, LES HÉSITATIONS ET L'EXPÉRIMENTATION}

Faisant le bilan des activités prenant appui sur les sentiers en 2016, l'Observatoire du développement durable de Corse pointe les risques et les opportunités liés à la revalorisation des chemins ruraux ${ }^{10}$ sur le plan écologique. Le constat pointe des ambiguïtés, la fréquentation apparaissant comme un risque pour la préservation des équilibres biologiques, alors que les tracés des chemins sont en parallèle présentés comme de possibles corridors écologiques et des supports pour le maintien d'un milieu naturel ouvert. De la même façon, des questions sont posées sur le plan social, entre ouverture du tourisme en arrière-saison, préservation de la qualité de vie des habitants et maîtrise de l'afflux de visiteurs, en particulier en termes de gestion des déchets ou de retraitement des eaux usées (Maupertuis, 2016).

Si les acteurs institutionnels et politiques conçoivent les sentiers comme des piliers de l'aménagement et de la valorisation des milieux périurbains et ruraux habités, les habitants entretiennent un rapport intime et identitaire aux lieux, pas forcément favorable à une ouverture à un trop large public exogène. Entre innovation territoriale et diversité des appréhensions, comment faire sens commun?

Il s'agit ici de questionner le sens des politiques et leur réception par les usagers à l'aune du concept de la justice environnementale, et de faire nôtre la position de Larrère posant que «l'environnement ne s'appréhende (...) pas seulement à l'aide de données objectives, il relève de l'étude du monde vécu » (Larrère, 2017: 14). Dans le cas balanin, malgré un affichage politique au sujet de l'environnement tourné vers le bien-être habitant, il persiste un sentiment d'injustice et des inégalités sont ressenties par les populations locales. Avant d'examiner comment cela se traduit, il convient de préciser notre positionnement quant au mouvement et à la théorisation de la justice environnementale.
L'historique autour de l'environmental justice est bien connu (Deldrève, 2015). Dans la lignée de ces travaux, nous adhérons à l'idée du caractère pluriel de la notion et à la nécessité d'aller au-delà d'une conception distributive, pour lui adjoindre les dimensions procédurales ou participatives, de reconnaissance et de capabilités (Deldrève et al., 2019: 2). Comme pour la question des inégalités, nous sommes pris entre deux terminologies. La justice «environnementale » envisagée «quand cette justice ne concerne que les rapports humains entre eux dans un contexte environnemental donné » et la justice «écologique» quand «on inclut dans les destinataires de la justice des vivants non humains, voire la Terre dans son ensemble » (Larrère, 2015: 5). Le cas des sentiers nous invite à adopter la posture «environnementale», en ce qu'elle est située et qu'elle traduit une politique d'aménagement valorisant des aménités naturelles avant tout destinées aux occupants humains de l'espace. Cependant, sans rejeter ce cadre important pour comprendre la dynamique institutionnelle en particulier, nous émettons l'hypothèse que les mobilisations sociales en Balagne opèrent un tournant assimilable au mouvement de justice écologique tel que l'a défini Schlosberg (2012). Élargissant la communauté de justice à l'ensemble des composantes des milieux, la justice écologique est alors à concevoir comme « une justice entre les hommes et la nature» (Lejeune, 2019: 5). Nous verrons que, même si le chemin d'une équité «hommes/ milieux » est encore long, l'expérience sensible défendue par Schlosberg occupe une place centrale dans les mobilisations et les processus participatifs. Cela nous amène à coupler ce courant avec l'«écologie des autres » de Descola (2011) qui met en avant l'existence de communautés sociales peuplées d'humains, d'animaux, de plantes ou encore de minéraux, pour une communauté de vie et de destin liée.

\section{Se confronter aux paradoxes: des initiatives multiples qui questionnent la cohérence et la durabilité des actions}

L'hétérogénéité des intervenants sur les sentiers de Balagne témoigne à la fois d'un dynamisme collectif et d'un éparpillement des orientations. Dans le cas balanin, le constat est qu'au-delà d'un intérêt partagé pour les sentiers, les réalisations sont le plus souvent fragmentées, chaque acteur agissant de façon isolée, sans concertation avec les autres protagonistes. Cela est vrai autant entre acteurs institutionnels qu'avec les acteurs sociaux. Cette atomisation ne relève pas d'un processus de méconnaissance. Le territoire est restreint, les gestionnaires se connaissent et connaissent souvent les personnes ressources parmi les résidents. L'absence d'échanges formalisés ${ }^{11}$ semble davantage tenir à des habitudes de travail différentes, chacun restant enfermé dans ses contraintes de gestion propres. Dans ce sens, les initiatives liées à une gouvernance locale partagée sont encore balbutiantes. Il en résulte un risque pour le territoire, celui d'initiatives contradictoires sur des portions de sentiers. De la même façon, le fait que pas moins de sept intervenants institutionnels ${ }^{12}$ agissent, peut entrainer une certaine cacophonie dans l'entretien des chemins. C'est ce que Blasquiet-Revol et al. (2017) ont constaté sur leurs terrains et que l'on a retrouvé en Balagne avec une gestion en régie directe pour certains réseaux et par délégation de mission de service public pour d'autres. Peuvent émerger ici ce que Laurent qualifie d'«inégalités dans la participation aux politiques publiques » (Laurent, 2017: 36). II y a en effet un accès inégal à l'information et au contenu même des actions engagées, «selon le statut social ou politique» (op. cit.). Si cela se règle relativement rapidement entre gestionnaires des espaces naturels, les usagers restent dubitatifs face aux changements parfois brusques dans les possibilités d'accès aux chemins ou aux moyens de déplacements autorisés ou non.

\footnotetext{
10 http://www.oddc.fr/modules.php?name=SimpleProfil\&op=showonedoc\&id=34\&mmg=9,528

11 Des contacts informels existent mais ne sont pas systématisés.

12 Ces intervenants sont: le Conservatoire du littoral, la DDTM, I'ONF, I'OEC, le PETR, les deux CC et les communes.
} 
Figure 4. Une diversité de signalétiques officielles

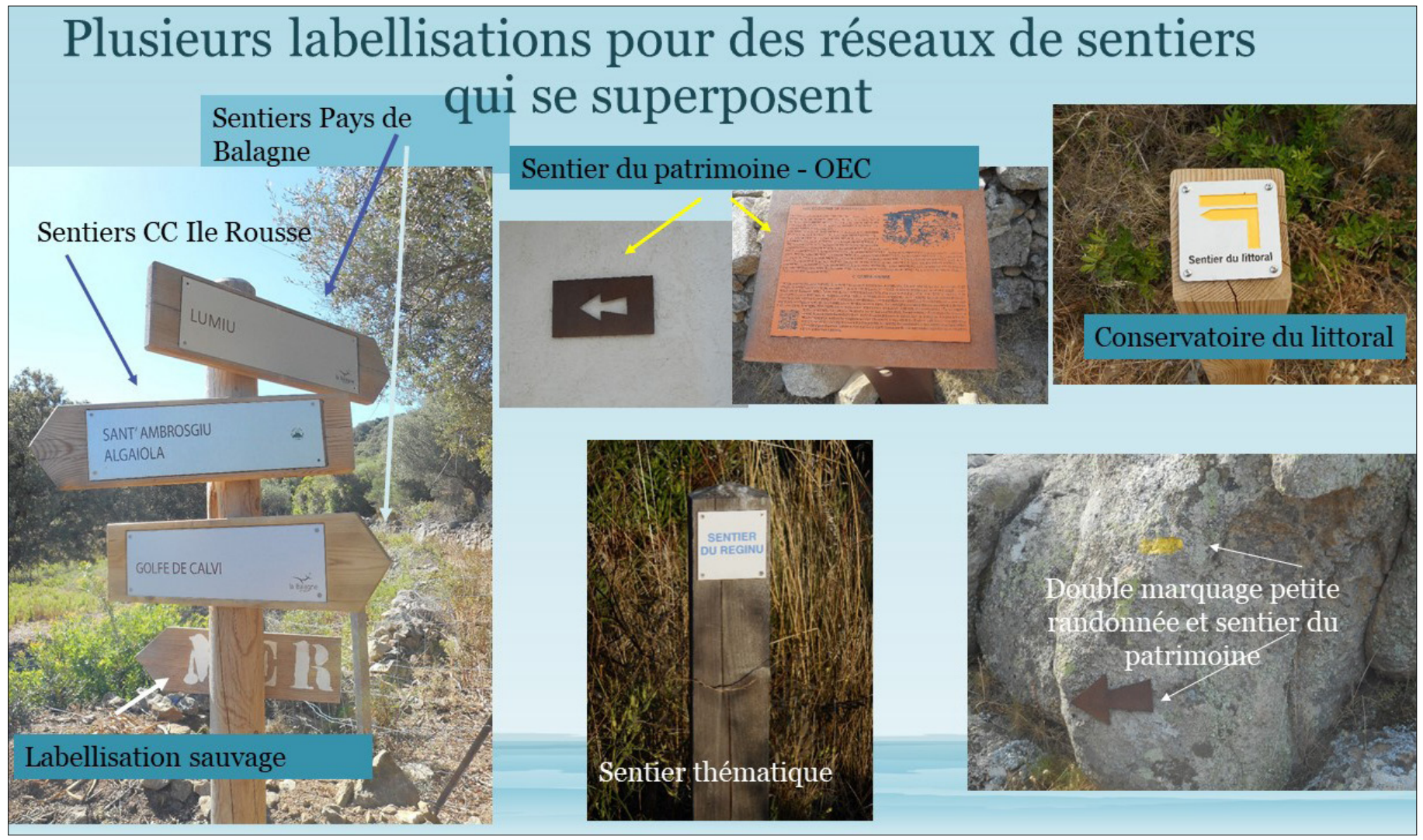

Source: Auteure

Figure 5. Des balisages anarchiques et peu identifiés

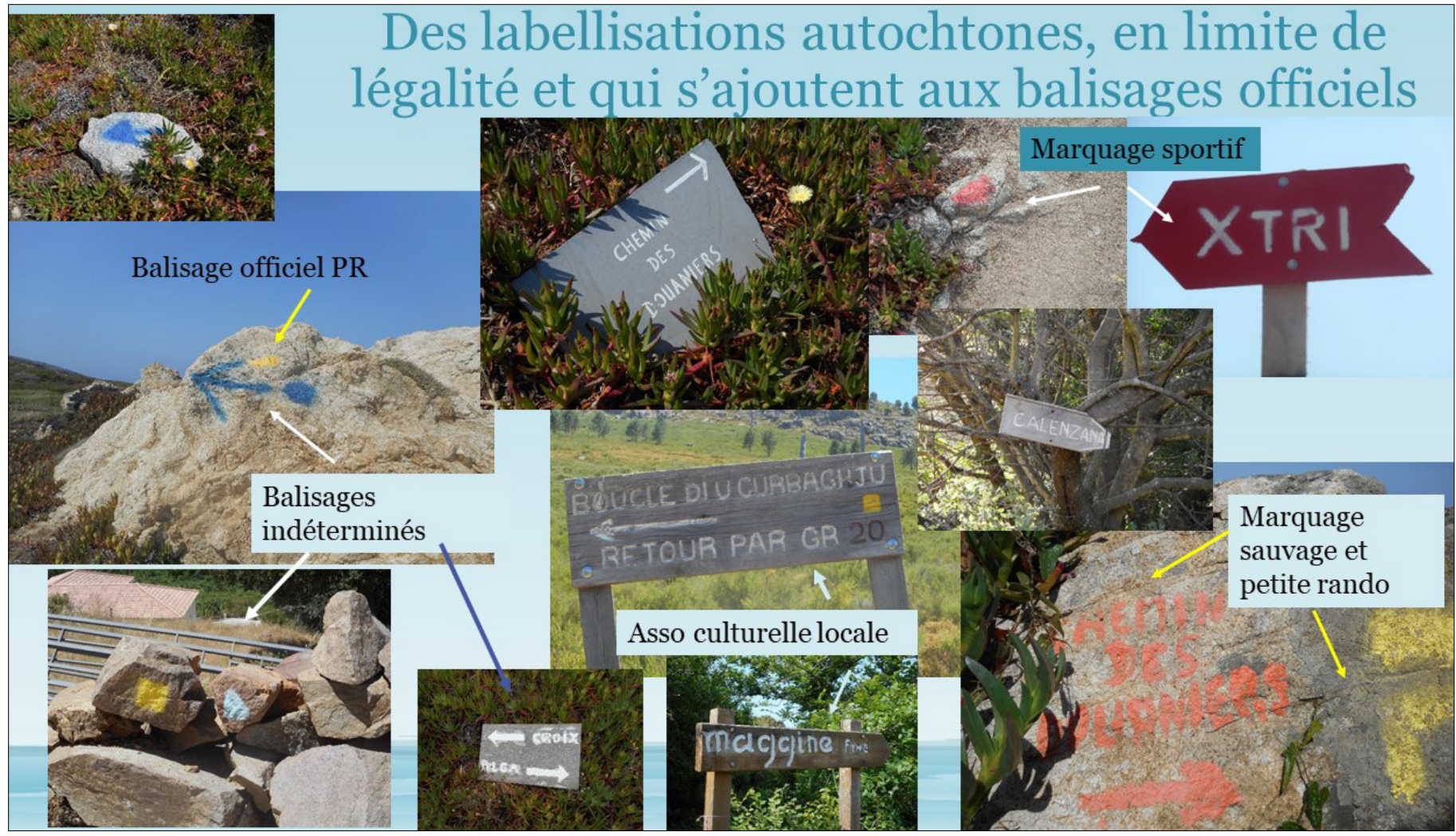


La diversité des parcours ouverts - par les institutionnels, les associations, des habitants isolés et des professionnels des loisirs de nature $^{13}$ - se traduit également par un certain «anarchisme», dénoncé par les acteurs eux-mêmes, dans la signalétique et le balisage. En effet, chaque institution a sa propre norme de balisage (figure 4), celle-ci se superposant avec des signalétiques associatives et des marquages non identifiés, faits à la bombe de peinture sous forme de traits, de flèches ou de points (figure 5). Ce surbalisage nuit à la cohérence de la politique engagée et entretient un flou sur les objectifs poursuivis et les paysages ainsi mis en avant.

Enfin, des conflits d'usages peuvent apparaître si les tracés ouverts à la fréquentation ne sont pas discutés avec les usagers du territoire, en particulier les agriculteurs et les éleveurs (figure 6), ainsi que les résidents dont I'habitation jouxte un sentier. Un détournement des sentiers piétons pour des usages motorisés est également constaté. Dans ces situations il y a un sentiment d'injustice vis-à-vis d'usages prédateurs non sanctionnés et les comportements non adaptés, même s'ils sont minoritaires, renforcent la défiance locale vis-àvis de l'extérieur, car c'est le touriste qui est pointé du doigt, ainsi que les opérateurs de loisirs de pleine nature. II est à noter que les plaintes des habitants dénoncent l'atteinte à leur cadre de vie dans une double dimension, sociale par l'atteinte faite à leur «tranquillité » et leurs habitudes, et écologique du fait des préjudices subis par les éléments du milieu naturel.

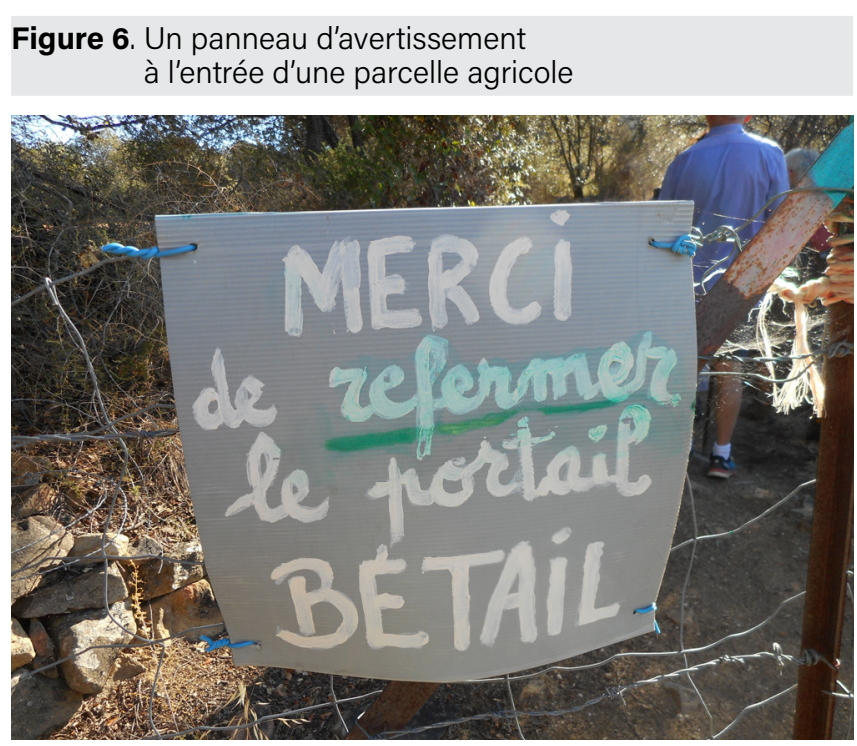

Source: Auteure

\section{Hésiter : un modèle de sentiers pris entre deux directions}

Tiraillé entre une économie touristique et l'encouragement au retour à une économie traditionnelle via l'agropastoralisme, ainsi qu'entre ouverture et préservation de l'identité, la Balagne semble à la recherche d'un équilibre, qu'elle teste via ses opérations autour des chemins ruraux.

Analysant les stratégies politiques régionales autour de l'aménagement et du développement de territoires iliens méditerranéens, Paoli et al. soulignent que «les conflits entre intérêt de préservation de l'environnement et intérêt économique lié au développement touristique des zones littorales constituent (...) un défi pour l'élaboration d'une démarche de planification territoriale » (Paoli et al., 2008: 159).
C'est ce qui se joue en Balagne. Les pourtours des villages et hameaux sont les lieux où il existe une densité importante de sentiers et là où les usages locaux sont aussi les plus importants. C'est donc avec ces cheminements «du quotidien» que se joue l'enjeu d'un développement rétro-littoral écologique et culturellement en phase avec l'identité locale. C'est aussi dans ce contexte que la politique d'aménagement doit être réfléchie pour impulser un basculement des touristes vers l'intérieur du territoire, perçu comme rééquilibrant avec la frange littorale, tant écologiquement, économiquement que socialement. Pour autant, il est compliqué de trouver une politique commune et cohérente à toute l'île (Fazi, 2010) ainsi qu'à la microrégion, présentant des caractéristiques locales différenciées entre pieves, ces anciennes circonscriptions administratives puis religieuses corses qui délimitaient les communautés.

Les élus et les techniciens qui ont mis en place les réseaux de sentiers tentent aujourd'hui de faire sens à la fois de façon pragmatique et écologique, en menant de front une politique tournée vers les habitants permanents et vers les visiteurs occasionnels. Siau souligne que « rares sont les projets de territoires qui se fondent sur ce que les habitants considèrent comme déterminant (...) plus rare encore sont les territoires qui choisissent de fonder leur politique touristique sur des bases identitaires» (Siau, 2007: 3-4). La Balagne innove donc en la matière.

La création de sentiers à vocation touristique et de loisirs a un impact, encore à évaluer, sur le plan environnemental. Si elle permet d'ouvrir les milieux et d'empêcher la progression du couvert forestier vers la mer $^{14}$, elle constitue une multiplication de portes d'entrées pour la circulation d'espèces invasives et un apport de déchets laissés par les randonneurs sur leur passage. S'il n'existe pas d'étude officielle quant à ces risques, ils sont régulièrement repris dans les propos des usagers. Comme Claeys-Meckdade \& Jacqué (2000) I'ont montré en milieu rural, s'il y a en pratique des usages proches de la nature partagés entre visiteurs et locaux comme la promenade, en réalité les significations accordées sont spécifiques. En Balagne les propos insistent sur la continuité entre les humains et leur milieu de vie. La nature de proximité apparait comme un prolongement «naturel» des habitants. Enfin, bien que les discours publics insistent sur la mise à disposition des chemins pour le bien-être des résidents en priorité, cela ne vient pas régler la question du prix du foncier et de l'accession à la propriété sur le littoral, qui se pose notamment pour les jeunes îliens. En effet, les terrains constructibles en bordure littorale sont souvent investis pour de la construction de logements à vocation touristique. Le prix élevé de l'immobilier, justifié par «la fonction touristique [qui] valorise le foncier insulaire» (Martinetti, 2007: 31), empêche les personnes aux revenus modestes - ce qui est fréquent en Corse, comme le souligne Fabiani, avec $20 \%$ de la population vivant sous le seuil de pauvreté - d'acheter ou de louer et donc de vivre en zone littorale. Les hausses de prix sont visibles à présent également en montagne et posent une vraie question quant aux possibilités offertes aux résidents permanents de se loger et surtout de pouvoir exercer leur liberté de choix quant aux lieux d'installation. Ce sont ici les capabilités (Sen, 2010) qui sont remises en cause. Cette situation foncière a des conséquences économiques, sociales et écologiques, qui nous amènent sur le terrain de la justice écologique. En effet, un arbitrage doit être fait entre la préservation de terres à vocation agricole et naturelle et leur mise en constructibilité (Tafani et al., 2012). Il y a ici un paradoxe, ou un effort supplémentaire à fournir par les pouvoirs publics. La ligne officielle du développement corse est celle d'un développement soutenable, d'une relocalisation des productions et d'une volonté de permettre aux jeunes de «vivre et travailler au pays». Cela reste cependant

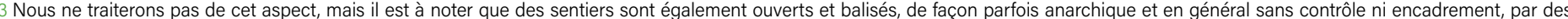

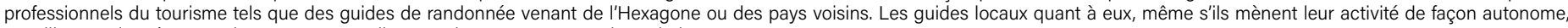
travaillent sur les réseaux existants et sont en lien avec les autres acteurs du territoire.

14 Voir données du Conservatoire botanique national de Corse.
} 
difficile et la préservation de terres non constructibles passe encore dans la majorité des cas par une préemption via le Conservatoire du littoral. Dans ce sens, les sentiers commencent à être saisis, pas forcément comme des remparts contre l'artificialisation des milieux, mais comme des moyens de préserver l'identité rurale des lieux et les composantes non humaines qui vont avec.

\section{Expérimenter: le sentier, un bien commun? Formes d'attachement et gestion collective comme innovations territoriales}

Formes d'attachement aux lieux, dynamique collective et innovation L'attachement manifesté envers des composantes de l'espace de vie se traduit de différentes façons. Concernant les chemins, il se manifeste par la pratique, la connaissance et la relation affective qui se noue. Cela se concrétise par des mobilisations collectives pour préserver les sentiers, les entretenir, ou par des pratiques plus individuelles mais non moins significatives d'appropriation. II ne s'agit pas d'une appropriation comme «droit d'usus et abusus sur une composante du monde» mais comme un lien d'interdépendance entre milieu et vivants qui positionne les humains comme «usufruitiers ou, dans certaines conditions, des garants. (...) Dans un tel cas, l'appropriation irait des milieux vers les humains, et non l'inverse» (Descola, 2015: 20). Les formes d'attachement apparaissent dans ce cas comme des innovations, car elles redéfinissent les relations au monde et engagent un rééquilibrage des échanges entre humains et non humains.

Les usages sociaux des sentiers de Balagne se déclinent le long d'un gradient allant de la déambulation contemplative à la performance sportive. Balades collectives ou solitaires, observations naturalistes et paysagères, cueillettes, marches sportives et trails, chacun imprime dans l'espace un rapport particulier aux lieux. Nous sommes ici dans « des formes de réappropriation active d'espaces communs » qui fondent la participation aux politiques environnementales de façon plus fondamentale que le simple «accès aux instances de décision » (Larrère, 2017: 26). La pratique quotidienne ou hebdomadaire, de proximité, permet alors de nouer des liens forts à l'espace, de le rendre «habitable» et par là de s'y projeter dans la durée (Mathieu, 2016). La relation « intime » créée peut être gage d'engagement écologique et social en enclenchant une «communion» avec les espèces (Kohn, 2017), un sentiment de devoir réciproque. Cette familiarité (Berroir et al., 2017) témoigne de l'importance du «sensible» pour comprendre l'engagement envers un territoire. Le proche, spatialement et symboliquement, devient alors un argument pour participer à la vie du territoire et décider des orientations de développement à prendre. Cela nous invite à élargir les modes de participation à la décision publique pour envisager la place de l'expérience sensible dans les processus participatifs (Schlosberg, 2012; Lejeune, 2019). Si les engagements habitants ne mettent pas explicitement en avant une justice pour le non humain, les discours engagent toujours un «nous» qui dépasse la seule communauté humaine. Il est possible de déceler dans les paroles et dans les actes, une dimension animiste qui confère une personnalité à tous les éléments du milieu avec lesquels l'humain entre en interaction ainsi qu'une dimension totémiste qui réaffirme l'attachement au lieu et l'origine commune qu'auraient tous les vivants et non vivants situés dans l'espace de vie de la communauté.

Comme l'ont souligné Fleury \& Prévot (2017), le contact avec la nature se fait par la cognition, les apprentissages et les expériences concrètes. II relève à la fois de l'expérience individuelle et collective ainsi que des échanges générés. Les pratiques habitantes peuvent donc alimenter les savoirs et participer à une forme de gouvernance écologique locale du territoire en faisant un lien entre savoir scien- tifique et savoir populaire (Moscovici, 2002). Les «croyances», les expériences sensibles aux lieux sont à considérer de façon complémentaire aux savoirs établis (Augagneur, 2017) et ont leur place dans les politiques d'aménagement, aux côtés de mesures plus techniques.

En Balagne, il est possible de postuler alors que, même si la valorisation des sentiers répond à un objectif institutionnel de développement territorial, les pratiques et l'engagement habitants constituent des interstices permettant de dépasser une logique marchande pour produire du sens, de l'identification et donc enclencher une gestion durable et innovante car elle redistribue la parole et confère à tous une compétence.

Revenir à une gestion collective de "communs » naturels et culturels via les chemins

Même si la dynamique autour des sentiers en Balagne se fait le plus souvent "en ordre dispersé», on voit émerger un sens commun entre les acteurs et des objectifs partagés entre usagers-habitants et gestionnaires. Les chemins de Corse appartiennent à l'histoire des lieux, ils sont ancrés dans la mémoire collective et ont structuré les modes de vie et les sociabilités (Meistersheim, 2006). Il existe en Corse une coutume d'entraide entre habitants, qui se manifeste par des «coups de main » face à une tâche lourde à accomplir. Ces Chjamà ${ }^{15}$ sont des réunions informelles, afin de participer à une action pour la communauté villageoise. C'était autrefois une forme de gestion collective d'espaces identifiés comme "communs», dont l'ensemble de la population pouvait jouir et donc qu'elle avait le devoir de sauvegarder. Plusieurs manifestations actuelles sur les sentiers peuvent être rapprochées de ces Chjama: lutte contre les espèces invasives sur le sentier littoral, participation des habitants au débroussaillage de chemins, notamment. L'encouragement à ce type de mobilisations peut être analysé comme une manière de dépasser les appropriations individuelles et de contourner les dispositifs de propriétés privées qui peu à peu ont remis en cause le «concernement» collectif (Augagneur, 2017). L'ordonnance de 1959 relative à la voirie des collectivités locales a en effet induit un processus de privatisation des chemins et contribué à la perte du sens collectif (Etcheverria, 1997). Réinstaurer une gestion partagée en permettant aux usagers d'agir concrètement sur et pour les chemins permet de refaire le lien défait aux lieux et d'ériger le sentier comme bien commun. La question des communs renvoie alors ici «aux modes d'être ensemble plutôt qu'à une qualité propre à ce qui est aussi utilisé en commun » (Larrère, 2017: 27).

La participation élargie à l'identification et à l'entretien des chemins peut également permettre de faire valoir l'environnement comme cause commune. II a été montré que concernant les questions écologiques, une «gouvernance territoriale endogène» était indispensable pour enclencher une dynamique vertueuse sur les plans environnemental et social et donc que l'échelon local - élargi aux habitants - était primordial. II s'agit d'établir de «nouvelles modalités de production de l'intérêt général » (Petrella et al., cité par Beuret \& Cadoret, 2011). En Balagne, l'intégration des visions et attentes des habitants aux processus de l'action publique locale montre une gouvernance innovante car elle favorise un équilibre entre besoins endogènes et exigences exogènes. Cela permet aussi de ne pas se limiter aux besoins économiques mais d'envisager une gestion plus large, incluant des facteurs culturels et environnementaux. La coconstuction - qui reste encore à bâtir - est indispensable dans cette situation et celle-ci doit se faire dans l'interaction, sur le mode du dialogue mais aussi de la confrontation (Larrère, 2015). 
Berroir, S. et al. (2017). Mobilité au quotidien et ancrage local dans les

Sur notre terrain d'étude, les habitants mobilisés ne représentent pas forcément la majorité des résidents. S'il n'est pas possible d'en dresser un profil type, la diversité l'emportant sur l'homogénéité, ils ont cependant une identité tournée vers l'engagement culturel et écologique la plupart du temps. Ils constituent donc une minorité qui souhaite agir. Pour autant, la recherche de légitimité de leur parole et de leurs actions n'est pas vaine et, à la suite de Moscovici et d'Augagneur, nous postulons que l'innovation territoriale se joue ici, dans «des allers-retours entre la délibération scientifique et l'expérience ordinaire », qui font émerger et évoluer les représentations sociales, et dans le dialogue avec une minorité ouvrant «des possibilités d'actions, de transformations et de créations » (Augagneur, 2017: 314). Comme Mathieu (2016: 574), nous pensons que «ce passage par l'individu est une étape nécessaire pour clarifier, aujourd'hui, ce que veut dire rapports à la nature». L'enquête a montré l'influence des pratiques sociales sur l'action politique par la prise en compte, encore à conforter, des usages locaux, la concertation émergente avec les acteurs et la transposition d'initiatives populaires dans des procédures techniques, pour la restauration patrimoniale des chemins notamment. La réhabilitation des sentiers, pilotée aujourd'hui par le Pays de Balagne et les intercommunalités, s'est inspirée d'une initiative populaire. C'est une association créée en 2001 et qui existe toujours, l'Association de réhabilitation des sentiers municipaux de la Balagne (ARSM), fondée par des habitants, qui a, la première, eu l'idée de rouvrir les sentiers, par une démarche d'insertion sociale de personnes éloignées de l'emploi. II a fallu près de vingt ans pour que cette démarche, longtemps restée isolée, aboutisse à une prise en compte des savoirs, des pratiques et des (bonnes) volontés habitantes par les acteurs gestionnaires des espaces naturels.

La dynamique des sentiers peut être analysée comme une innovation locale, pas tant du fait des politiques d'aménagement, même s'il faut souligner la dynamique de soutenabilité au fondement des initiatives, mais surtout en raison de la prise en compte des mouvements sociaux. II semble se manifester ici un mouvement de justice écologique par l'engagement habitant et la mise en œuvre de capabilités qui affirment le droit à choisir, à se faire entendre et à participer pour modeler son lieu de vie. Les attachements à la communauté biotique et abiotique locale qui traduisent une relation sensible au vivant, ont alors toute leur place dans les processus de décision politique. La dynamique couplée des habitants et des gestionnaires du territoire, même si elle peut répondre à des injonctions et des désirs différents, nous oriente vers une «écologie de la réconciliation » (Rosenweig, 2003). II s'agit de pouvoir s'affranchir des intérêts individuels, ou de les transcender, pour prendre soin de soi et des autres, d'affirmer l'importance d'un bien-être du vivant et non exclusivement humain. C'est inventer une façon alternative d'«habiter le monde» (Mathieu, 2016). C'est aussi l'idée que préserver la diversité biologique avec les populations peut engendrer davantage de justice sociale. La réussite des politiques écologiques et de l'aménagement des territoires passe alors par un impératif: «prendre en considération les contraintes qui pèsent sur la population locale concernée, mais aussi ses représentations, ses savoirs et ses savoir-faire» (Larrère \& Larrère, 2009: 212).

Les risques, les incertitudes et les conflits potentiels et réels autour des sentiers sont ainsi à envisager comme des indicateurs de changement et des producteurs d'innovation.

\section{BIBLIOGRAPHIE}

Augagneur, F. (2017). Écologie et psychologie sociale, in C. Fleury \& A-C. Prévot (dir.), Le souci de la nature. Paris: CNRS, 305-318. espaces périurbains. Annales de géographie 713(1) : 31-55.

Beuret, J-E. \& A. Cadoret. (2011). Une gouvernance territoriale endogène de l'environnement: contours et enjeux. Géographie, économie, société 13(4) : 363-386

Blasquiet-Revol, H., L. Amblard, O. Aznar \& C. Déprés (2017). Organisation de l'entretien de la voirie locale dans les territoires ruraux. Le cas de trois communes du Puy-de-Dôme. Économie rurale [En ligne] 360.

Bourg, D. (2020). Inégalités sociales et écologiques. Revue de l'OFCE 165(1): 21-34.

Boutelet, M. (1983). Un régime juridique pour les chemins de randonnée pédestre. Revue juridique de l'Environnement 4 : 291-323

Chamboredon, J-C. (1985). La «naturalisation » de la campagne: une autre manière de cultiver les «simples »?, in C. Cadoret (dir.), Protection de la nature, histoire et idéologie. De la nature à l'environnement. Paris: L'harmattan, 138-151.

Claeys-Mekdade, C. \& M. Jacqué. (2000). Le massif forestier de la Clape: nature de proximité et nature pittoresque? Etude de fréquentation auprès des usagers. Forêt méditerranéenne XXI(1) : 19-24

Claeys, C., A. Hérat, C. Barthélémy \& V. Deldrève (2016). Quand les Calanques deviennent Parc National: disputes autour de la définition et de la répartition des efforts environnementaux et urbains induits. Norois 238-239: 71-84.

Dechézelles, S. \& M. Olive (2016). Introduction. Lieux familiers, lieux disputés - dynamiques des mobilisations localisées. Norois 238239: 7-21.

Descola, P. (2011). L'écologie des autres. L'anthropologie et la question de la nature. Versailles: Quae.

Descola, P. (2015). Humain, trop humain. Esprit 12: 8-22.

Deldrève, V. (2015). Pour une sociologie des inégalités environnementales. Bruxelles: PIE Peter Lang.

Deldrève, V., N. Lewis, S. Moreau \& K. Reynolds (2019). Les nouveaux chantiers de la justice environnementale. Introduction. VertigO [En ligne] 19(1). https://doi.org/10.4000/vertigo.24863.

Deldrève, V. (2020). La fabrique des inégalités environnementales en France. Revue de l'OFCE 165(1): 117-144.

Etcheverria, O. (1997). Le chemin rural, nouvelle vitrine des campagnes? Strates [En ligne] 9.

Fabiani, J-L. (1982). Quand la chasse populaire devient un sport. La redéfinition sociale d'un loisir traditionnel. Études rurales 87-88: 5180.

Fabiani, J-L. (2018). Sociologie de la Corse. Paris: La Découverte.

Fazi, A. (2010). Les stratégies d'aménagement régional en Corse: espoirs et désillusions, in M-A. Maupertuis (dir.), La Corse et le développement durable. Ajaccio: Albiana/CNRS,169-176.

Fleury, C. \& A-C. Prévot. (2017). Le souci de la nature. Paris: CNRS.

Ginelli, L. (2017). Jeux de nature, natures en jeu. Des loisirs aux prises avec l'écologisation des sociétés. Bruxelles: PIE Peter Lang.

Haschar-Noé, N. (2009). L'aménagement des chemins de randonnée: un instrument d'identification et de «gouvernance» territoriales. Espaces et Sociétés 138(3) : 115-133.

Kalaora, B. (1993). Le musée vert. Radiographie du loisir en forêt. Paris: L'Harmattan.

Kalaora, B. (2010). Rivages en devenir: Des horizons pour le Conservatoire du littoral. Paris: La Documentation Française. 
Kohn, E. (2017). Comment pensent les forêts. Paris: Zones Sensibles.

Larrère, R. \& C. Larrère. (2009). Du principe de naturalité à la gestion de la biodiversité, in R. Larrère, B. Lizet, M. Berlan-Darqué (dir.), Histoire des parcs nationaux. Paris: Quae, 205-222.

Larrère, C. (2015). Environnement: ne pas ignorer les conflits. Revue Projet, 347(4) : 20-24.

Larrère, C. (dir) (2017). Les inégalités environnementales. Paris: Presses universitaires de France.

Laurent, E. (2017). Mesurer et réduire les inégalités environnementales en France, in C. Larrère (dir.), Les inégalités environnementales. Paris: Presses universitaires de France, 29-51.

Le Breton, D. (2000). Éloge de la marche. Paris: Métailié.

Lefevre, M. (2001). Écologie et géopolitique en Corse. Hérodote $100(1): 32-54$.

Lejeune, C. (2019). Justice sociale et durabilité, la rencontre est-elle possible? Portée politique de l'expérience vécue des injustices écologiques. VertigO [En ligne] 19(1), consulté le 29 août 2020. https:// doi.org/10.4000/vertigo.24217.

Lenclud. G. (2012). En Corse, une société en mosaïque. Paris: MSH.

Logossah, K. \& M-A. Maupertuis (2007). La spécialisation touristique des petites économies insulaires en développement est-elle une voie de croissance durable? Revue d'Économie Régionale \& Urbaine 2007/1: 35-55.

Londei, D. (2020). «L'intelligence de la nature»: une nouvelle Babel ou enfin un vrai dialogue entre les espèces vivantes? Les Carnets du Cediscor [En ligne] 15.

Martinetti, J. (2007). Les tourments du tourisme sur lî̂le de beauté. Hérodote 127(4) : 29-46.

Mathieu, N. (2016). Modes d'habiter, in A. Choné, I. Hajek \& P. Hamman (dir.), Guide des humanités environnementales. Lille: Presses universitaires du Septentrion, 567-582

Maupertuis, M-A., M. Isola \& X. Pieri. (2016). Vivre sur un littoral touristique. Attentes citoyennes et enjeux méthodologiques d'un indice synthétique de qualité de vie, in S. Robert \& H. Melin (dir.), Habiter le littoral, enjeux contemporains. Aix-en-Provence: Presses Universitaires d'Aix-Marseille, 447-464.

Meistersheim, A. (2006). Le malentendu. Entre imaginaire insulaire et imaginaire continental. Ethnologie française 36(3): 503-508.

Melin, H. \& M. Poli. (2021). Comment habiter un littoral insulaire touristique? Pratiques et histoires locales autour de sentiers pédestres comme preuves de l'investissement ilien. Norois à paraître, automne 2021.

Melin, H. (2015). La pratique des sentiers périurbains de Balagne (Corse) par les habitants: marcher pour réinventer sa vi(II)e. Environnement Urbain / Urban Environment [En ligne], 2015/9.

Mignotte, A. (2002). Vers une gestion concertée et participative des réseaux de sentiers et des espaces naturels protégés. Revue de géographie alpine 90(2): 45-59.

Moscovici, S. (2002). Réenchanter la nature. Paris: Éditions de l'Aube.

Paoli, J-C., A. Fiori \& R. Melot. (2008). L'aménagement du littoral à l'épreuve de la décentralisation. Conflits et concertation en Corse et Sardaigne. Pôle Sud 28(1): 143-165.

Perrin-Malterre, C. (2014). Identité touristique des stations de sports d'hiver de moyenne montagne et recompositions territoriales. Annales de géographie 697(3): 935-955.

Roseinweig, M. (2003). Win-win ecology. Oxford: Oxford University Press.
Sahuc, P. (2010). Chemins périurbains: aménités vécues et enjeux réels. Natures Sciences Sociétés 18(2):147-157.

Sansot, P. (2000). Chemins aux vents. Paris: Payot/Rivages.

Schlosberg, D. (2012). Climate Justice and Capabilities: A Framework for Adaptation Policy. Ethics \& International Affairs 26(4) : 445-461.

Sen, A. (2010). L'idée de justice. Paris: Seuil.

Siau, V. (2007). Sports de nature et attractivité des territoires. POUR 194(2): 13-20.

Tafani, C. (2010). Littoral corse: entre préservation de la nature et urbanisation, quelle place pour les terres agricoles? Méditerranée 115: 79-91.

Tafani, C., M-A. Maupertuis \& X. Pieri. (2012). Impact du tourisme sur le prix du foncier agricole en Corse: la durabilité de l'agriculture littorale en question, in B. Mésini (dir.), Aménagement durable des territoires méditerranéens. Aix-en-Provence: Presses Universitaires d'Aix-Marseille, 73-87.

Theys, J. (2007). Pourquoi les préoccupations sociales et environnementales s'ignorent-elles mutuellement? Un essai d'interprétation à partir du thème des inégalités écologiques, in P. Cornut, T. Bauler \& E. Zaccaï (dir.), Environnement et inégalités sociales. Bruxelles: Éditions de l'Université de Bruxelles, 23-36.

Torre, A. et al. (2006). Conflits et tensions autour des usages de l'espace dans les territoires ruraux et périurbains. Le cas de six zones géographiques françaises. Revue d'Économie Régionale \& Urbaine 2006/3: 415-453.

Viard, J. (2012). Penser la nature. La Tour-d'Aigues: Éditions de l'Aube.

Vuillamier, M. (2019). Spanu en Balagne, histoire de la mutation d'un domaine ecclésiastique en territoire touristique ( $X \mathrm{I}^{\mathrm{e}} \mathrm{-} X \mathrm{X} \mathrm{e}^{\mathrm{e}}$ siècle). Thèse de doctorat d'histoire, Université de Corte, décembre 2019. 